\title{
Cholera in Niger Republic: An Analysis of National Surveillance Data,
}

\section{$1991-2015$}

\author{
Lawaly Maman Manzo, ${ }^{1, *}$ Aichatou Moumouni, ${ }^{1}$ Idi Issa, ${ }^{2}$ Ali Amadou, ${ }^{1}$ Jibir Zanguina, ${ }^{1}$ Dan Dano \\ Ibrahim, ${ }^{1}$ Halima Boubacar Mainassara, ${ }^{2}$ and Sani Ousmane ${ }^{1}$ \\ ${ }_{1}^{1}$ Laboratory of Bacteriology-Virology/ National Reference Laboratory of Cholera and Other Gastroenteritis Diseases/Centre de Recherche Médicale et Sanitaire (CERMES)/ \\ International Network of Institutes Pasteur, BP 10887 - 634 Bd de la Nation, YNo34, Niamey, Niger \\ ${ }^{2}$ Epidemiology Unit of Infectious Diseases / Centre de Recherche Médicale et Sanitaire (CERMES)/ International Network of Institutes Pasteur, BP 10887 - 634 Bd de la Nation, \\ YNo34, Niamey, Niger \\ "Corresponding author: Lawaly Maman Manzo, Laboratory of Bacteriology-Virology/ National Reference Laboratory of Cholera and Other Gastroenteritis Diseases/ Centre de \\ Recherche Médicale et Sanitaire (CERMES)/ International Network of Institutes Pasteur, BP 10887- 634 Bd de la Nation, YNo34, Niamey, Niger, E-mail: \\ lawaly.manzo@cermes.org
}

Received 2016 December 21; Accepted 2016 December 28.

\begin{abstract}
Background: Cholera is an infectious disease caused by a gram negative bacteria, Vibrio cholerae. It was found for the first time in Niger during year 1970 and continues to occur as an issue of major public health importance.

Objectives: This study aimed at more accurately defining the burden of cholera in Niger.

Methods: Cholera surveillance data from the direction of epidemy surveillance and response reported to the Niger ministry of public health and the world health organization were reviewed and analyzed to determine trends in cholera disease.

Results: Of Niger's 8 regions or states, 7 were very active in reporting cases each year between 1991 and 2015. Globally, cholera cases and deaths were 26,835 and 1,430 , respectively (CFR $=5.3 \%$ ).

Conclusions: Taken together, the epidemiological trends of cholera in Niger showed annual increase in frequency and length of outbreaks during the 21 years under review. To successfully reduce the burden of cholera outbreaks in Niger, concerted efforts by the government and non-government organizations as development partners through longterm investments are urgently needed to strengthen preventive measures for early detection and confirmation of cases, followed by a well-coordinated, timely, and effective response.
\end{abstract}

Keywords: Africa, Niger, Epidemiology, Cholera, Outbreaks, Surveillance, Vibrio Cholerae

\section{Background}

Cholera is an enteric gastroenteritis disease caused by gram negative bacteria known as Vibrio cholerae, either type $\mathrm{O} 1$ or O139. The disease is considered among the leading causes of morbidity in Niger and can effect both children and adults. Cholera cases have been reported annually in many parts of the world. Cholera outbreaks have persisted for long in many African countries since 1970. In 1971, Niger experienced its first worst cholera outbreak with 9265 cases, including 2344 deaths (CFR\% = 25.3) (case fatality rate) (1). Since this disaster, cholera outbreaks have been reported annually, with the exception of a few years, in Niger.

Niger, situated in Western Africa, is an Inland subSaharan country devided to 8 geographical regions. Countries sharing boders with Niger include Nigeria and Benin (from the south side), Burkina Faso and Mali (from the west side), Algeria and Libya (from the north side), and Chad (from the east side) (2).

In Niger, 7 regions out of 8 have experienced most cholera outbreaks, with most affected areas positioned near Niger River (Dosso, Tillabery and Niamey), Lake Chad (Diffa) or fishing villages from Dosso, Tahoua, Maradi, and Zinder (3). In 2013, Didier B. et al., demonstrated the implication of various risk factors, which encourage the emergence of cholera epidemy in Niger (2). From this study, it was agreed that cholera frequently affected communities located along borders with neighboring countries (Nigeria, Tchad, Mali, and Benin).

In Niger, the Ministry of Public Health has enunciated programs for the elimination, eradication prevention, and control of important infectious diseases, including cholera, with support from development partners. In order to improve the quality of the surveillance systems across the country, Niger together with other African countries, adopted in 1998, the integrated disease surveillance and response (IDSR) strategy launched by the world health organization African regional office (WHO/AFRO) (4). Important decisions and engagements were taken by the government officials, and the country adapted IDSR for better

Copyright @ 2017, International Journal of Infection. This is an open-access article distributed under the terms of the Creative Commons Attribution-NonCommercial 4.0 International License (http://creativecommons.org/licenses/by-nc/4.0/) which permits copy and redistribute the material just in noncommercial usages, provided the original work is properly cited. 
management of most epidemics. Since the adoption and implementation of IDSR strategy across the country, most technical steps involved in the process of surveillance were strengthned.

This study reviewed and analyzed the epidemiological status of cholera in Niger on the basis of cholera surveillance data reported by DSRE (Directorate of Surveillance and Response to Epidemics) to the ministry of public health, the world health organization, and other development partners from 1991 to 2015, using annual DSRE reports, the outbreak bulletin, and weekly epidemiological reports from WHO.

\section{Objectives}

The present study was a comprehensive analysis of the national surveillance data to accurately define the incidence of cholera in Niger.

\section{Methods}

\subsection{Cholera Surveillance in Niger}

Niger has embraced a new IDSR strategy and has also introduced it in all regions using protocols developed by WHO and center of diseases control (CDC). The introduction of IDSR in Niger has encouraged the establishment of a national epidemiology division, DSRE, with the mission of epidemic management. The epidemiology division of the ministry of public health within the DSRE coordinates and conducts cholera and other gastroenteritis surveillance in Niger with support from the national reference laboratory (NRL) housed inside the 'Centre de Recherche Médicale et Sanitaire (CERMES)', a member of the Pasteur Institute network (2). Daily reports of suspected cases, identified by peripheral health centers or the 'integrated health center (IHC)', are compiled by district health personnel and then sent to the regional delegations of public health for diseases surveillance, which compile data as summary statistics and then send it to the national division of diseases surveillance and response and to the WHO.

Notification of most cases of cholera in Niger is made on the basis of clinical signs and symptoms and of geograpical locations detailed in the IDSR technical guidelines for standard case definition (4). Confirmed cases are those in whom Vibrio cholerae, Ogawa, serotype $\mathrm{O} 1$ has been isolated from their stool. Microbiological surveillance is pursued for the purpose of outbreak confirmation; usually, a representative number of stool specimens ( $\leq 10$ samples) were deducted from the total number of received ones for bacteriological analysis. At the end of the outbreak, universal testing of all the remaining nontested stool specimens of suspected cases received during the peak period was started up again. At district level, mainly rapid test or RDT, microscopy of stool and Gram staining were performed. Culture and/or molecular detection and typing by polymerase chain reaction (PCR) were performed at the national level, more precisely within MHRC/NRL.

\subsection{Laboratory Testing for Cholera}

The national reference laboratory for cholera and other gastroenteritis is the key and accredited national laboratory for suspect cholera cases confirmation in Niger. The laboratory is mandated to conduct various microbiological tests on collected samples at the beginning of an outbreak for clinical diagnosis confirmation and to confirm regression and/or end of an outbreak. Summary laboratory data were obtained from CERMES (2).

Stool and rectal swab specimens destined for microbiological testing are transported to the laboratory in CaryBlair transport media. All health centers across the country were provided with transport medium. A well-organized network for rapid transport of prepared specimen was implemented by DSRE in collaboration with private human traffic transportation mechanism to forward the specimen to DSRE then to CERMES within 36 hours. For the identification and isolation of $V$. cholerae strains, enriched and selective culture medium were used. Biochemical methods, which include the use of API2OE galery for enteric bacteria, Oxidase or Indole tests for identification of $V$. cholerae were also performed. Vibrio cholerae O1 polyvalent antiserum and the monovalent Ogawa and Inaba antisera were tested separately against the selected Vibrio cholera-positive isolate for strain and serotype confirmation by slide agglutination tests. A representative number of culture-positive strains is selected for polymerase chain reaction analysis for further molecular characterization of the isolated $V$. cholerae $\mathrm{O} 1$ to understand more about the circulating clones during a particular outbreak to help for vaccine strategy.

\subsection{Data Analysis}

National surveillance data for cholera reported to the Niger Ministry of Public Health and the WHO by the DSRE for years 1991 to 2015 were evaluated. From years 1991 to 2007 and 2008 to 2015, the total number of reported cholera cases and deaths were obtained from the WHOAfrican database and from the DSRE/ CERMES-national database, respectively. Incidence per 100,000 population and case fatality rate (CFR) for each year were determined through the analysis of the collected data. Demograpic 
data estimating the population number during a particular period were extracted from the 'national institute of statistics (NIS)', under the Niger Ministry of Planning. Laboratory data were extracted from the national reference laboratory for cholera and other gastroenteritis reports and analyzed. All analysis of the cholera surveillance data were performed with the Microsoft Excel Office, version 2013.

\section{Results}

\subsection{Epidemiology of Cholera}

From 1991 to 2015, a total of 26835 cases, including 1430 deaths, were announced to the Ministry of Public Health and the world health organization (overall CFR 5.3\%). The epidemiological evolution in cholera cases and variation in CFRs from year to year, are described in Figure 1.

Weekly epidemiological records from world health organization revealed a high frequency and distribution of cholera epidemics in Niger during 1991 to 2006, with 3238 cases in 1991, 3957 in 1996, 2178 in 2004, and 1232 in 2006 (1). Following a rapid decline in the number of cases in 2007, the country experienced another important resurgence of cholera cases in the regions (Diffa, Dosso, Maradi, Niamey, Tahoua, and Zinder) with less access to safe water and proper sanitation during the period from 2008 to 2015 (5). During this period, Niger Ministry of Public Health received a total of 12,643 reports of cholera cases, including 410 deaths, corresponding to an overall CFR of 3.2\% (Table 1). From January to early December 2012, Niger reported 5,285 cases including 108 deaths (CFR 2.04\%) (1). About $90 \%$ and $9 \%$ of reported cases were from the region of Tillabery and the region of Tahoua, respectively. While sporadic cases were reported in Dosso, Maradi, and Niamey. The peak cholera incidence during this period was 32.6 per 100,000 population (Table 1 ). Cholera cases reported during 2010 to 2013 (9,583 cases with 256 deaths, CFR 2.7\%) originated from the neighboring health districts referred as "Hotspots" for cholera entry in Niger and more frequently affected regions (Tahoua, Maradi and Zinder) that shared borders with Nigeria, the region of Dosso(sharing border with Nigeria and Benin) and of Diffa (sharing border with Nigeria and Tchad) (3). The region of Tillabery experienced the largest number of cases reported during this period (7057 cases). Ayorou, Tera, Tillabery, Kollo, and Gotheye considered as 'Hotspots' for cholera entry in Niger recorded more than $95 \%$ of the recorded cases in the country for these past 4 years. These localities are particularly around Niger River. Globally, a total of 14 districts out of 32 across the country were identified as 'Hotspots' for cholera by the ministry of public health (Figure 3) (2). Also, their safe water supply is relatively poor. However, in 2014, the total number of reported cases from the region of Tillabery (72 cases) was far less compared to those reported in other regions, such as Maradi (306 cases), Tahoua (572 cases), and Diffa (537 cases).

Between January and September, 2015, Niger reported 51 cases, including 4 deaths (CFR 7.84\%) $(5,6)$. The total number of reported cases by the DSRE/MSP from January $23^{\text {rd }}$ to $28^{\text {th }}$ was 25 cases with 22 from Kollo, including 4 deaths and only 3 from Tillabery, including no death. From January $1^{\text {st }}$ to $24^{\text {th }}, 26$ cases were notified in the region of Diffa. In Tillabery and Diffa, the principal cause of the cholera epidemy was the lack of safe water supply and hygenic conditions. Notified cases from Diffa comported a few imported cases from Nigeria, the neighboring country (5). However, despite the decrease noted in the total number of suspected cases in 2015, the CFR was high (7.8\%) compared to 2008 (7.2\%), 2010 (5.7\%), 2011 (2.5\%), 2012 (2.1\%), 2013 (2.53\%), and 2014 (3.9\%). Overall, since 2008, the national incidence kept decreasing progressively to its lowest level in 2015(0.3 per 100,000 population)(Table 1 ). Comprehensive illustrations and analysis of reported data of annual number of cholera cases, deaths, and CFR in Niger from 1991 to 2015 and by region affected in Niger from 1994 to 2013 are presented in Figure 1 and Figure 2, respectively.

\subsection{Laboratory Data}

Of the 12643 cases reported from 2008 to 2015, 1,152 specimens (9.1\%) were microbiologically confirmed at the National Reference Laboratory/CERMES (Table 2); 328 (48.7\%) were culture positive for $V$. cholerae O1. From these culture-positive specimens, 129 were tested for strains and serotype confirmation by Polymerase Chain Reaction (PCR). All the tested specimens by molecular methods were confirmed positive for $c t x B, r s t R, t c p A$, zot, ace, and $c t x A$ which are genes coding for $V$. cholerae 01 toxines.

\section{Discussion}

Review and analysis of the cholera surveillance data presented here demonstrated that cholera epidemy is dynamic in Niger. The general tendancy showed annual increase of the frequence and length of epidemics. All recorded outbreaks were confirmed to be due to V. cholerae O1 serotype Ogawa.

Significant decrease of the cholera incidence was observed in 2015. Niger experienced 9 of the largest cholera epidemics with number of reported cases being $>1000$, across successive years of outbreaks. There are several potential explanations for the presence and spread of cholera 


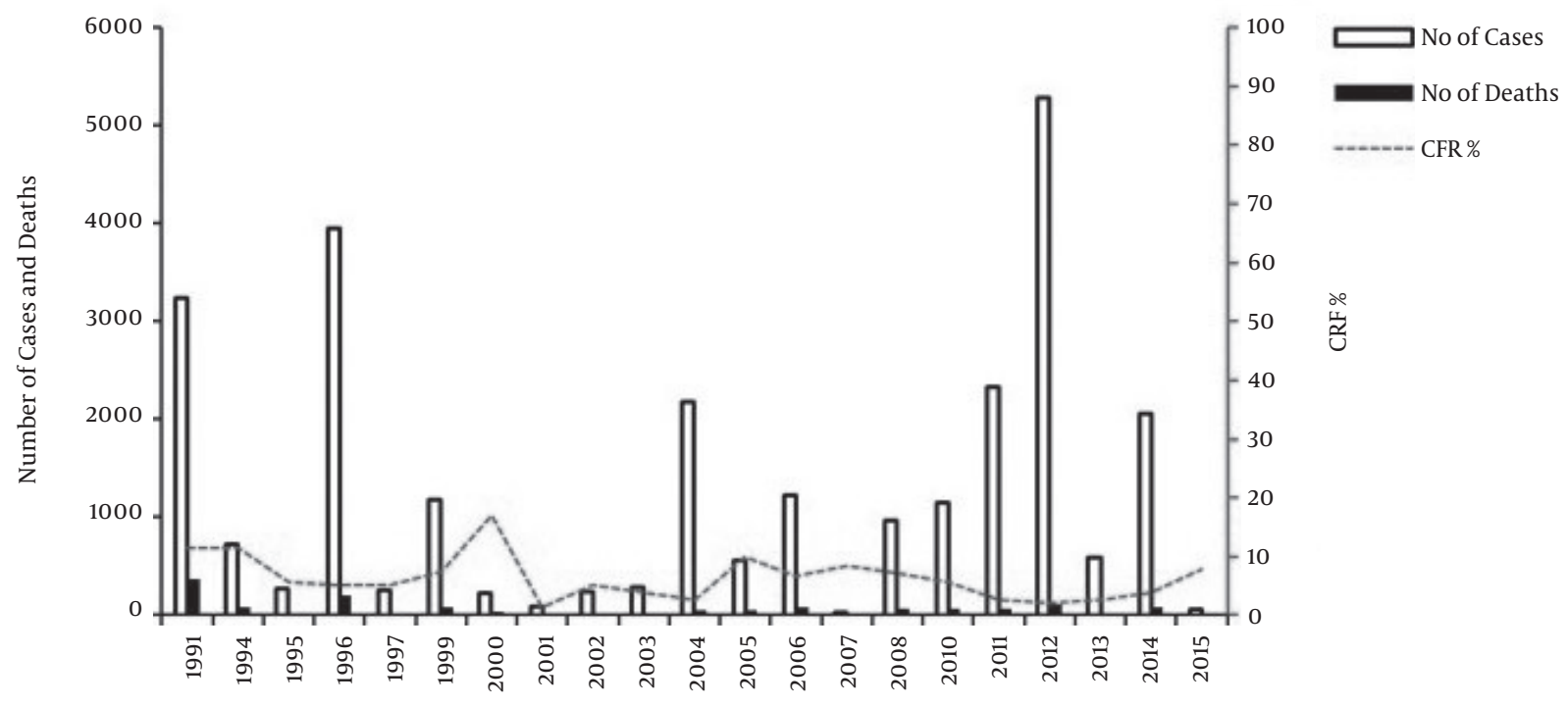

Year

Figure 1. Cholera Cases, Deaths and CFR, 1991 to 2015



Figure 2. Annual Number of Cholera Cases, Deaths and CFR Percentage by Region Affected in Niger, 1994 to 2013 (3)

in areas situated in the South of Niger, which includes regions such as Diffa, Dosso, Maradi, Tahoua, Tillaberi, Niamey, and Zinder. Areas across and surrounding the Niger River and the Lake Chad, respectively, have particularly experienced severe outbreaks (7, 8). From 2004 to 2014, Niger, Nigeria, and Chad, three countries bordering the Lake Chad Basin reported to WHO a total of 226,855 cholera cases and 5,711 deaths $(9,10)$. Riverside countries in the Niger River have also experienced cholera burden. Be- tween 1991 and 2008, 2011 and 2013, and 2015, the region of Tillabery, across the Niger river had the largest number of cholera cases $(3,5)$. In 2010 and 2014, the region of Diffa, near the Lake Chad Basin reported greater total number of cholera cases compared to those reported in other regions during the same period. Spread of cholera across Niger as a country has also benefited from human traffic along its main borders, in particular across borders with Nigeria, Chad, Benin, and Mali. The regions sharing borders with 



IDUSSA@CERMES.ORG

BENIN

Figure 3. Hotspots Districts for Cholera in Niger

Table 1. A Summary of Reported Number and Average Incidence per 100,000 Population of Notified Cases of Cholera Between 2008 and 2015

\begin{tabular}{|c|c|c|c|c|c|}
\hline Year & No. of Cases & No. of Deaths & Case Fatality ratio, \% & Population, Millions & Incidence, per 100,000 \\
\hline 2008 & 950 & 70 & 7.3 & 14.5 & 6.5 \\
\hline 2010 & 1272 & 76 & 5.9 & 15.2 & 8.3 \\
\hline 2011 & 2434 & 57 & 2.3 & 15.7 & 15.5 \\
\hline 2012 & 5285 & 108 & 2.0 & 16.2 & 32.6 \\
\hline 2014 & 2059 & 80 & 3.8 & 17.8 & 11.5 \\
\hline 2015 & 51 & 4 & 7.8 & 18 & 0.3 \\
\hline Total & 12643 & 410 & 3.2 & & \\
\hline
\end{tabular}

Abbreviation: No, Number.

Nigeria (10), recorded up to $30 \%$ of the cholera cases. While the regions of Dosso and Diffaappeared were less affected, yet could be areas with high risks. In general, the country has endured cross-border outbreaks coming from Nigeria (2008, 2010, and 2011) and from Mali (1996, 2004, and 2006) (3). In Niger, cholera spreads in the region of Lake Chad
Basin to the Chad (2004 and 2010) and across the Niger River to the Benin (2006 and 2008), Mali (2011) and less frequently to Burkina Faso (2011 and 2012) (11-13). Following 21 years of outbreaks reported from across the country in 1991 to 2012 and 2014, there was a significant decrease in cholera cases reported in 2013 and 2015. 
Table 2. Laboratory Results for Reported Cholera Cases, Laboratoire National de Reference (LNR), Niger, 2008 to 2015

\begin{tabular}{|c|c|c|c|c|c|c|c|}
\hline \multirow[t]{2}{*}{ Year } & \multirow{2}{*}{$\begin{array}{l}\text { Suspected Cholera } \\
\text { Cases Reported, No. }\end{array}$} & \multicolumn{2}{|c|}{ Conventional Culture Method } & \multicolumn{2}{|c|}{ Molecular Method (PCR) } & \multicolumn{2}{|c|}{ Serotype, No. (\%) } \\
\hline & & $\begin{array}{l}\text { Specimens tested, } \\
\text { No. (\%) }\end{array}$ & $\begin{array}{c}\text { Specimens culture } \\
\text { positive for cholera, } \\
\text { No. }(\%)\end{array}$ & $\begin{array}{l}\text { Specimens tested, } \\
\text { No. (\%) }\end{array}$ & $\begin{array}{c}\text { Specimens PCR } \\
\text { positive for cholera, } \\
\text { No. (\%) }\end{array}$ & Ogawa & Inaba \\
\hline 2008 & 950 & $65(6.8)$ & $21(32.3)$ & $4(19)$ & $4(100)$ & $21(100)$ & 0 \\
\hline 2010 & 1272 & $130(10.2)$ & $57(43.8)$ & $49(85.9)$ & $49(100)$ & $57(100)$ & 0 \\
\hline 2011 & 2434 & $254(10.4)$ & $141(55.5)$ & $66(46.8)$ & $66(100)$ & $141(100)$ & 0 \\
\hline 2012 & 5285 & $356(6.7)$ & $169(47.5)$ & $5(2.9)$ & $5(100)$ & $169(100)$ & 0 \\
\hline 2013 & 592 & $64(10.8)$ & $18(28.1)$ & - & - & $18(100)$ & 0 \\
\hline 2014 & 2059 & $258(12.5)$ & $77(29.8)$ & $5(2.9)$ & $5(100)$ & $77(100)$ & 0 \\
\hline 2015 & 51 & $25(49)$ & $10(40)$ & - & - & $10(100)$ & 0 \\
\hline Overall & 12643 & $1152(9.1)$ & $328(48.7)$ & $129(39.3)$ & $129(100)$ & $328(100)$ & 0 \\
\hline
\end{tabular}

Abbreviation: No, Number.

The quality of cholera case management significantly depends on CFR, an important indicator that demonstrates the success or not of various strategies developed against a given cholera outbreak (14). Considering the years under review, the cholera CFR in Niger remains far higher than the $1 \%$ considered by the WHO as a standard upper limit $(15,16)$, and CFRs also varied considerably from outbreak to outbreak. An underestimation of the actual rate of deaths during outbreaks could be strongly hypothesized to be due to the timid reporting of cases and or deaths to the DSRE then the ministry.

Cholera epidemics resurgence in Niger began in 2013, with a comprehensive study with the ambition to describe various risk factors contributing to the emergence of cholera in Niger, and to identify principal routes of cholera entry into the country and thus formulate recommendations for better global management of this public health concern. Data analysis revealed that all cholera outbreaks that had happened in Niger started first in the neighboring health districts sharing borders with Nigeria, Chad, and Mali. These neighboring health districts were though referred as "Hotspots" for cholera entry in Niger. Frequently affected health districts were situated across the Niger River (Tera, Tillabery, Kollo, Niamey, and Gaya), at the border with Nigeria (Madarounfa, Birni N'konni, Madaoua, and Maradi) and at the border with Chad (Diffa) (2).

This study has significantly contributed in compiling knowledge about risk factors, which certainly do accelerate the rapid spread of the cholera in the country and in identifying for the first time, the principal route of cholera penetration in Niger, through its neighbors. These evi- dences have warned the government and other development partners such as WHO, UNICEF, UNHCR, and UNOCHA, and NGOs in taking actions for a successful fight to reduce the burden of cholera epidemy in the country. Important measures through the IDSR strategies should be reinforced for containing the spread of cholera.

The present study had several limitations. The DSRE's annual reports were used from 2002 to 2013 to estimate the burden of cholera during this period. Annual statistical reports of cholera from 1991 to 2001, 2014 and 2015 were not available, and therefore epidemiological bulletins from the WHO were used in order to complete the analysis. Microbiological confirmation of most cases is unknown. Most laboratories throughout the country lack the capacity to isolate the bacterial pathogen, and this may have certainly influenced the cases distribution. Many of the regions and/or villages may have reported certain cases of diarrhea to the national surveillance system not due to cholera, despite having the same risk factors as those that detection or laboratory confirmation have been done. These incoherences can certainly lead to overestimation or underestimation of cholera lethality. Globally, reporting of outbreaks and cholera cases in Niger lack promptitude and completeness with significant delays in publication.

Cholera has been dynamic in Niger. Controlling the outbreak will require the consideration of various limiting factors that contribute to the rapid spread of the disease. Cholera hotspots situated along the boader with Nigeria and Niger are ways of intensive exchange with neighboring countries (Mali, Benin, Nigeria, and Tchad) $(17,18)$. Prioritized strategic actions in the districts, included early detection, community-based surveillance, ac- 
tivities near boaders, specific activities of preparativeness and response to outbreaks. Programs encouraging Longterm access to water, for hygiene and sanitization should be prioritized and realized in districts regularly affected by increasing incubation period during eipidemy. An integrated study, WASH-Epidemiology could be an opportunity to define durable responses through programs in cholera hotspots.

\subsection{Conclusion}

Taken together, the present study has provided substantial knowledge about the cholera burden in Niger.

\section{Acknowledgments}

The authors thank the ministry of public health, 'directorate of surveillance and response to epidemics', the 'Centre de Recherche Médicale et Sanitaire (CERMES)' and the 'national institute of statistics', and both internal and external partners involved in cholera surveillance in Niger, for their support.

\section{Footnotes}

Authors' Contribution: Lawaly Maman Manzo conceived, designed, analyzed and wrote the manuscript. Lawaly Maman Manzo, Aichatou Moumouni, Ali Amadou, Jibir Zanguina, Dan Dano Ibrahim and Sani Ousmane participated in collecting data and in revising the manuscript. Halima Boubacar Mainassara and Idi Issa participated in providing the map and in revising the manuscript.

Conflict of Interests: The authors declare that there is no conflict of interest regarding the publication of this manuscript.

Funding/Support: This work was supported by the 'Centre de Recherche Médicale et Sanitaire (CERMES)' through the national reference laboratory for Cholera and other gastroenteritis diseases.

\section{References}

1. World Health Organization. Global task force on cholera control

2. Ministere de la Sante Publique . Plan strategique multisectoriel d'elimination du cholera au niger, 2015-2019 [In French]. 2015

3. UNICEF. Cholera epidemiology and response factsheet Niger. 2013

4. World Health Organization . Technical guidelines for integrated disease surveillannce and response in the African region. 2nd ed. ; 2010.

5. OCHA . Diffa: évolution de la situation humanitaire Personnes déplacées à Zinder [In French]. 2015 :1-5.

6. Kivu S. Cholera Outbreak in Central and West Africa: 2015 Regional Update - Week 46. 2015

7. Rebaudet S, Sudre B, Faucher B, Piarroux R. Environmental determinants of cholera outbreaks in inland Africa: a systematic review of main transmission foci and propagation routes.J Infect Dis. 2013;208 Suppl 1:S46-54. doi: 10.1093/infdis/jit195. [PubMed: 24101645].

8. Bompangue Nkoko D, Giraudoux P, Plisnier PD, Tinda AM, Piarroux M, Sudre B, et al. Dynamics of cholera outbreaks in Great Lakes region of Africa, 1978-2008. Emerg Infect Dis. 2011;17(11):2026-34. doi: 10.3201/eid1711.110170. [PubMed: 22099090].

9. UNICEF . Cholera outbreak in the West and Central Africa: Regional update, 2014 - WEEK 52. 2015

10. UNICEF. Cholera epidemiology and response factsheet nigeria. 2014

11. World Health Organization. Report of first meeting of the global task force for cholera control. Jun, 2014

12. Rebaudet S, Sudre B, Faucher B, Piarroux R. Cholera in coastal Africa: a systematic review of its heterogeneous environmental determinants. J Infect Dis. 2013;208 Suppl 1:S98-106. doi:10.1093/infdis/jit202. [PubMed: 24101653].

13. Konate I. Epidemies de cholera en afrique de l ' ouest de 1995 a 2004. 2006.

14. Antoine B, Guy J, Anne RNS. Prise en charge d ' une épidémie de choléra [In French]. Paris: Médecins Sans Frontiere; 1995. p.107.

15. Djomassi LD, Gessner BD, Andze GO, Mballa GA. National surveillance data on the epidemiology of cholera in Cameroon.J Infect Dis. 2013;208 Suppl 1:S92-7. doi: 10.1093/infdis/jit197. [PubMed: 24101652].

16. Mutonga D, Langat D, Mwangi D, Tonui J, Njeru M, Abade A, et al. National surveillance data on the epidemiology of cholera in Kenya, 1997-2010. J Infect Dis. 2013;208 Suppl 1:S55-61. doi: 10.1093/infdis/jit201. [PubMed: 24101646].

17. World Health Organization/Regional Office for Africa . Integrated disease surveillance quarterly bulletin. May 2015

18. Oger PY, Sudre B. Eau - Hygiène - Assainissement et Epidémiologie du choléra dans les pays du bassin du lac Tchad [In French]. UNICEF. 2011. 